\title{
To Preserve Human Health, It is Time to Stop Feeding Antibiotics to Farm Animals in the USA and Other Countries
}

\section{Arnold L Demain*}

Research Institute for Scientists Emeriti (R.I.S.E.), Drew University, Madison, New Jersey 07940, USA

Keywords: Antibiotics; Animal growth; Nosocomial infections; Human diseases; Antibiotic discovery; Antibiotic resistance

\section{Editorial}

Antibiotic discovery was a major factor in the increase in life expectancy in the USA from 47 years in 1900 to 74 for males and 80 for women in 2000. However, resistance development in pathogens has become a major problem. Infectious diseases cause $20 \%$ of all deaths in the world and are the leading cause of death in children under the age of five. They are the second leading cause of death in the world (13 million deaths) and number three in developed nations. In the USA, 2-2.5 million people are infected each year with antibiotic-resistant bacteria. Hospital-acquired infections in the USA cost the health care system over $\$ 20$ billion per year and lead to more than eight million additional days in the hospital. In Europe, the numbers of antibiotic-resistant infections are 400,000 per year resulting in 25,000 deaths. Much of the problem deals with hospital-acquired (nosocomial) infections. Each year, there are about two million nosocomial infections in the USA, resulting in 90,000 to 200,000 deaths. About half of these are caused by Staphylococcus aureus. Others are mainly due to infections with Clostridium difficile and Pseudomonas aeruginosa. These three organisms produce septicemia, especially in old and immunecompromised patients. Resistant bacteria which have developed over the years are generally uninhibited by most commercial antibiotics today. For example, some enterococci are resistant to all known antibiotics. Other organisms that are not normally virulent can infect immune-compromised patients. Great concern exists about resistance development among Gram-positive pathogens, such as methicillinresistant bacteria including $S$. aureus, i.e., the well-known MRSA. The incidence of MRSA infections in U.S. hospitals went from 127,000 in 1999 to 278,000 in 2005 and to 360,000 by 2011. During that time, U.S. deaths from MRSA increased from 11,000 to 19,000. MRSA attacks 150,000 people per year in Europe and kills about the same number of Europeans as Americans. Another serious problem involves clinical isolates of penicillin-resistant Streptococcus pneumoniae, the most common cause of bacterial pneumonia. It increased in the USA from 1987 to 1992 by 60 -fold. It causes $1,200,000$ cases in the USA each year and 7,000 deaths. Other serious nosocomial infections involve the intestinal bacterium Klebsiella pneumoniae causing pneumonia, blood infections, infections in newborns and intensive-care patients. It can be cured by the beta-lactam carbapenem antibiotics, such as imipenem and meropenem, but carbapenem-resistance is developing rapidly, especially in hospitals. In some countries, half of the patients die from carbapenem-resistant infections. The number of beta-lactamases discovered in clinical isolates is enormous, i.e., about 1300 . The most dangerous are the extended spectrum beta-lactamases that hydrolyze most penicillins and cephalosporins. The carbapenases inactivate virtually all beta-lactam drugs. Hospital-acquired pathogens produce multiple beta-lactamases, as high as eight such enzymes per strain. Carbapenem-resistant Enterobacteriaceae increased four-fold in the USA from 2003 to 2013 with a mortality rate of $50 \%$.

Gastrointestinal infections killed 17,000 people in the USA in
2007 as compared to 7,000 in 1999. Two-thirds of the deaths from gastrointestinal infections are caused by $C$. difficile, mainly in nursing homes, hospitals, doctors' offices and clinics. $83 \%$ of the deaths occurred in people older than 65 years of age. A second cause of gastrointestinal infections is the norovirus, occurring in hospitals, prisons, dormitories and cruise ships. Norovirus causes 20 million infections per year in the USA and 800 deaths. Urinary tract infections by Escherichia coli were once easily cured by use of fluoroquinolines but resistance is now widespread.

Enteric infections caused by bacteria, viruses or fungi are the fifth leading cause of death from disease worldwide. $70 \%$ of the cases are food-borne. In the USA, 3,000 deaths occur from enteric infections each year. Around the world, 1.5 billion cases of diarrheal disease occur annually, killing 1.8 to 2.2 million people, mainly children. Those under five years of age are most at risk. Vibrio cholerae is a major cause of acute watery diarrhea. Serogroups 01 and 0139 of $V$. cholerae cause five million cases and 100,000 deaths per year.

C. difficile is a major problem in and out of hospitals and the numbers of such infections are increasing. Cases in U.S. hospitals increased from 139,000 in the year 2000 to 250,000 in 2014. Three million cases occurred outside of hospitals. Mortality rates quadrupled from 1999 to 2014 in the USA. At least one-third of affected patients experience a recurrence within one month of treatment. C. difficile has surpassed MRSA as the leading hospital-acquired infection in the USA. In 2004, a more virulent and drug-resistant strain evolved, producing two toxins causing problems in cells lining the intestine. The cost of $C$. difficile infections in the USA has exceeded $\$ 1$ billion per year.

A major problem, especially in hospitals, is the formation of biofilms growing on wounds, scar tissue, medical implants (joint prostheses, heart valves, spinal instruments, vascular prosthetic grafts). Biofilms are important in infections of cystic fibrosis, otitis media, endocarditis and other urinary tract infections. Bacteria present in biofilms are very resistant to antibiotics that normally would inhibit the individual species in the free state. In the UK, about $30 \%$ of the patients over 60 years of age and $41 \%$ of those over 90 die within 30 days.

Tuberculosis is mainly caused by Mycobacterium tuberculosis, but also by Mycobacterium bovis, Mycobacterium caprae, Mycobacterium microti, Mycobacterium pinnipedii, Mycobacterium canetti, and

*Corresponding author: Arnold L Demain, Research Fellow, Research Institute for Scientists Emeriti (R.I.S.E.), Drew University, Madison, New Jersey 07940, USA, Tel: (1)-973-408-3937; E-mail: ademain@drew.edu

Received August 10, 2015; Accepted August 11, 2015; Published August 18 2015

Citation: Demain AL (2015) To Preserve Human Health, It is Time to Stop Feeding Antibiotics to Farm Animals in the USA and Other Countries. J Microb Biochem Technol 7: e123. doi:10.4172/1948-5948.1000e123

Copyright: @ 2015 Demain AL. This is an open-access article distributed under the terms of the Creative Commons Attribution License, which permits unrestricted use, distribution, and reproduction in any medium, provided the original author and source are credited. 
Mycobacterium africanum. Two billion people each year around the globe have tuberculosis and 8 to 13 million come down each year with lung infections due to tuberculosis. India has the major problem with about $40 \%$ of its population infected. Globally, 1.4 to 2.6 million people dies each year. 100,000 to 630,000 of these deaths are due to multidrug-resistant $M$. tuberculosis (MDR-TB). The Bacillus CalmetteGuerin (BCG) live vaccine, first used in 1921, is $80 \%$ effective in protecting young children against tuberculosis but it has little effect for adolescents and young adults. It does not prevent TB infections in lungs. Vaccines developed to prevent tuberculosis have failed, although there are twelve new ones in clinical trials. Among HIV-positive immune-compromised patients, TB is the leading cause of death. Over 3 million people globally have HIV, one million residing in the USA. In 2008, there were 3.3 million people with HIV around the world, of which 2 million died. In the USA that year, one million people had HIV and 50,000 new cases occurred.

The gonorrhea bacterium, Neisseria gonorrhea, infects more than 100 million people around the world. Although it was treatable in the 1970s with penicillin, it has become resistant to penicillin and is becoming resistant to $3^{\text {rd }}$ generation oral cephalosporins. Tetracycline was once effective but today, $80 \%$ of gonorrhea-producing bacteria are resistant to tetracycline.

Fungal infections are very serious, especially when they involve immune-compromised patients, e.g., systemic fungal infections are responsible for the death of $50 \%$ of leukemia patients. Fungal infections doubled from the 1980s to the 1990s with bloodstream infections increasing five-fold and an observed mortality of $55 \%$. There is an increasing incidence of candidiasis, cryptococcosis and aspergillosis, especially in AIDS patients. Failure rates in aspergillosis exceed $60 \%$. Fungal infections often occur after transplant operations at the following rates: $5 \%$ for kidney, $15-35 \%$ for heart and lung, up to $40 \%$ for liver transplants. $80 \%$ of these events are caused by species of Candida and Aspergillus. Pulmonary aspergillosis is the main factor involved in death of recipients of bone marrow transplants and Pneumocystis carinii is the major cause of death in U.S. and European patients with AIDS. Fungal infections cause $40 \%$ of nosocomial infections. Candida infections affect 60,000 to 70,000 patients per year in the USA and kill between 3,000 and 11,000 Americans annually due to nosocomial candidemia.

All of the above problems are intensified by the lack of introduction of new antibiotics. The glory years were from the 1940s until the end of the 1960s. The number of anti-infective investigational new drugs (INDs) declined by $50 \%$ from the 1960 s to the late 1980 s. Between 1983 and 1987, the FDA approved 16 new antibiotics. In the next four years, the number dropped to 14. Between 2008 and 2012, only two new antibiotics were approved. Of 95 new pharmaceuticals approved by the FDA from 2010 to 2012, only one was a new systemic antibiotic. In the 1950s, there were 35 companies working on antibiotics. By 1990, 18 pharmaceutical companies were working on antibiotic discovery, but by 2013 , there were only four. One of the problems is the difficulty in finding a promising drug. For every 100,000 compounds tested in animals, only ten enter clinical trials, and only one is commercialized. It takes 15 years for discovery, preclinical testing, phases 1, 2 and 3 of clinical testing, and one year to obtain the new drug application. This costs about $\$ 1.2-1.6$ billion dollars per drug.
Despite all the above tragedies, feeding of antibiotics to farm animals in the USA and in many other countries continues, and is mainly done to promote animal growth. Today, $80 \%$ of total antibiotic use is for animals. Thirty different antibiotics have been used in food and water fed to animals. It is expected that there will be an increase in worldwide use of antibiotics in food animals of $67 \%$ between 2010 and 2030. This use of antibiotics in agriculture as growth promotants increases bacterial resistance to antibiotics. Antibiotic resistantmicrobes cause 2 million infections annually in the USA and kill thousands of Americans each year. This application of antibiotics is the leading cause of antibiotic-resistance in the USA. In 2012, over 16,000 tons of antibiotics were fed to animals. By 2030, this unwise activity of using antibiotics for growth promotion of farm animals could amount to 100,000 tons of antimicrobials per year. This practice is prevalent mainly in China, followed by the USA, Brazil, India and Mexico. Unfortunately, it selects for resistant bacteria that spread into the human population. China grows and consumes 500 million pigs per year. These animals excrete over 600 billion kilograms of manure each year containing bacteria with resistance genes, as well as thousands of tons of antibiotics which end up in lakes and other waterways. China uses 150,000 to 200,000 tons of antibiotics for humans and farm animals each year, about ten times as much as in the USA. Of antibiotics sold in the USA, $73 \%$ go into animals. Of those antibiotics produced in the USA, amounting to 50 million pounds annually, $40 \%$ are used in animals. In the USA, 28 million pounds of antibiotics were used for farm animals in 2009 and this number increased to 32 million pounds in $2012.60 \%$ of the antibiotics fed to animals are important for human health. As far back as 1977, the U.S. Food and Drug Administration proposed the elimination of tetracycline, chlortetracycline and penicillin from animal feeds but this was not supported by the U.S. Congress. On the other hand, The European Union has been phasing out use of antibiotics for animal growth since the late 1990s, with Denmark enacting a complete ban in the year 2000. Later, Europe banned antibiotic use in farm animals and observed that emergence and spread of resistant bacteria has been controlled and even reversed. It is time that the USA and many other countries wake up and realize the importance of this threat to human health. 